\title{
Understanding structures at the base of the solar corona - polar plumes
}

\author{
A. H. Gabriel ${ }^{1}$, F. Bely-Dubau ${ }^{2}$, E. Tison ${ }^{1}$ and L. Abbo ${ }^{3}$ \\ ${ }^{1}$ Institut d'Astrophysique Spatial, Université Paris Sud-11, \\ 91405 Orsay Cedex, France \\ email: gabriel@ias.fr \\ ${ }^{2}$ Observatoire de la Côte d'Azur, BP4229, 06304 Nice Cedex, France \\ ${ }^{3}$ Osservatorio Astronomico di Torino, Pino Torinese 10025 - Italy
}

\begin{abstract}
Recent work on coronal polar plumes (Gabriel et al. 2003, 2005) has aimed at determining the outflow velocity in plume and interplume regions, using the Doppler dimming technique on oxygen VI observations by SUMER and UVCS on SOHO. By comparing observations of SOHO/EIT with plume modelling, we show that the major part of plumes is the result of chance alignments along the line-of-sight of small enhancements in intensity. This confirms the so-called curtain model. These plumes can be attributed to reconnection activity along the boundaries of supergranule cells. A second population of plumes has a lower abundance and arises from surface bright points having a particular magnetic configuration. New observations using the Hinode/EIS spectrometer are in progress, with the aim of providing further insight for this model.
\end{abstract}

Keywords. Sun: corona, solar wind

The plumes that we are addressing here are those seen extending out to $0.5 R_{\odot}$ or more beyond the solar limb, when looking in spectral regions emitting at temperatures around $1 \mathrm{MK}$ or rather less. They are best observed within polar coronal holes and during a solar minimum configuration. They are well observed from SOHO by SUMER and EIT in the oxygen VI $1032 \AA$ and the Fe IX $171 \AA$ band, respectively. Here we concentrate on the EIT observations, since these exist in long synoptic series of images. Existing measurements of physical properties (Wilhelm et al. 1998; Wilhelm 2006) show the temperature to be around $0.8 \mathrm{MK}$ and the density of order $10^{8} \mathrm{~cm}^{-3}$. Gabriel et al. (2003), Gabriel et al. (2005) and Teriaca et al. (2003) used Doppler dimming techniques (Noci, Kohl \& Withbroe 1987) to measure the outflow velocity. Velocities were found to be higher than in the background corona up to $1.5 R_{\odot}$, then falling below coronal values at greater heights. We believe that there are two different kind of plume phenomena:

- Beam plumes, quasi cylindrical structures rooted in bright magnetic structures on the disk.

- Curtain plumes, faint sheet-like structures visible only when seen edge-on.

Our contribution aims to present evidence for this distinction. Many other workers in the field have assumed that plumes are always of the beam plume type.

The most convincing evidence that plumes are not always (even, not usually) of the beam type comes from simply looking at images of a sufficient number of plumes. Fig. 1 shows four successive images of the south solar pole at 7 day intervals, equivalent to $90^{\circ}$ rotation between each image. Almost all of the plumes, irrespective of the viewing direction, appear to start at the solar limb. On the other hand, in the plane of the sky, plumes appear to be evenly distributed over angles of $\pm 20^{\circ}$ from the solar axis. If these were beam plumes, we would therefore expect to see about half of them originating on 


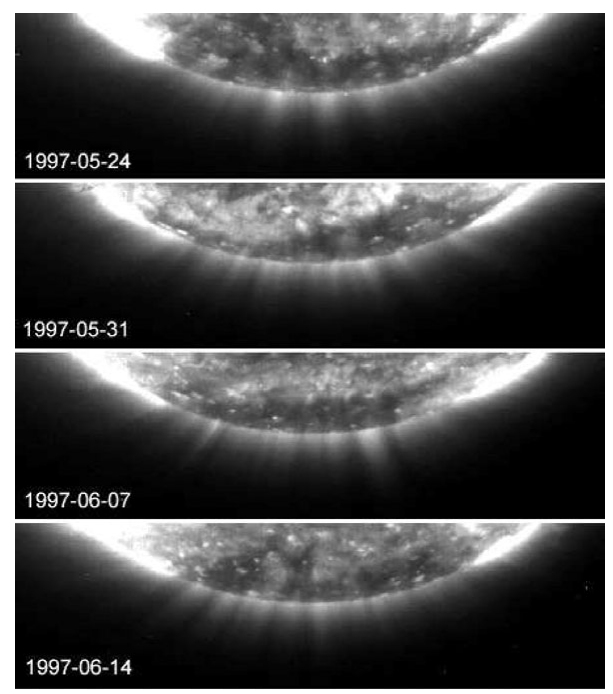

Figure 1. Four EIT images of the south polar coronal hole in Fe IX radiation, taken at 7 day intervals.

the visible solar disk. This is not the case. All of the plumes appear to have their origin behind the solar disk, which clearly cannot be the case. We find, and will demonstrate in what follows, that the majority of these plumes are curtain plumes, in which the observed brightness arises through a substantial line-of-sight integration, involving path-lengths of
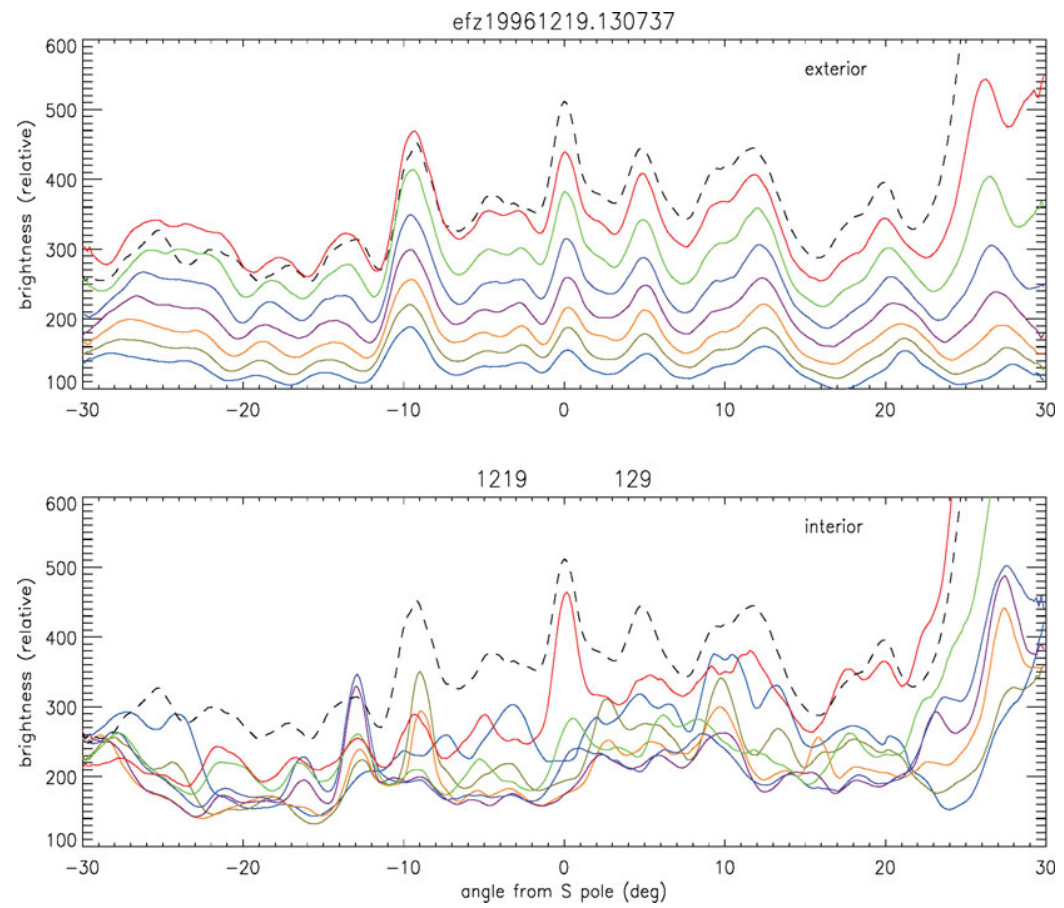

Figure 2. A series of limb scans of a polar coronal hole. The curves in the top panel are outside the limb and the curves in the bottom panel inside. The black dashed curve is shown on both plots and it is at a radius of $1.01 R_{\odot}$, close to the radius of maximum brightness. The other curves are stepping outwards (top panel) and inwards (bottom panel) in intervals of $0.01 R_{\odot}$. 
the order $0.5 R_{\odot}$ or greater. Some beam plumes are also present, but are in the minority and usually fainter.

Because of concerns about the misleading impression by images having a limited dynamic range, as well as by problems of visual perception, we further develop this argument in a more quantitative form. Fig. 2 shows the results for the brightness structure of one of these limb images. The curves in the upper panel show clearly the plumes, decreasing in brightness with radial distance. The curves in the lower panel show how this above-limb plume structure decreases rapidly as we pass onto the disk. An obvious plume does rarely maintain its brightness profile for more than 2 further steps or $0.02 R_{\odot}$ (equivalent to an absolute height of $0.99 R_{\odot}$ ), giving way to a different structure arising from brightness patterns on the disk. It should be remembered that a real beam plume, on the front side of the Sun is expected to increase in brightness as we pass from the limb on to the disk.

To extend our quantitative evaluation, we produce simulated models of what would be expected from the two proposed plume geometries, beam plumes and curtain plumes. The plume material is known to be optically thin, and to fall off in height with a scaleheight not too different from the quiet coronal hole corona. The calculation is therefore
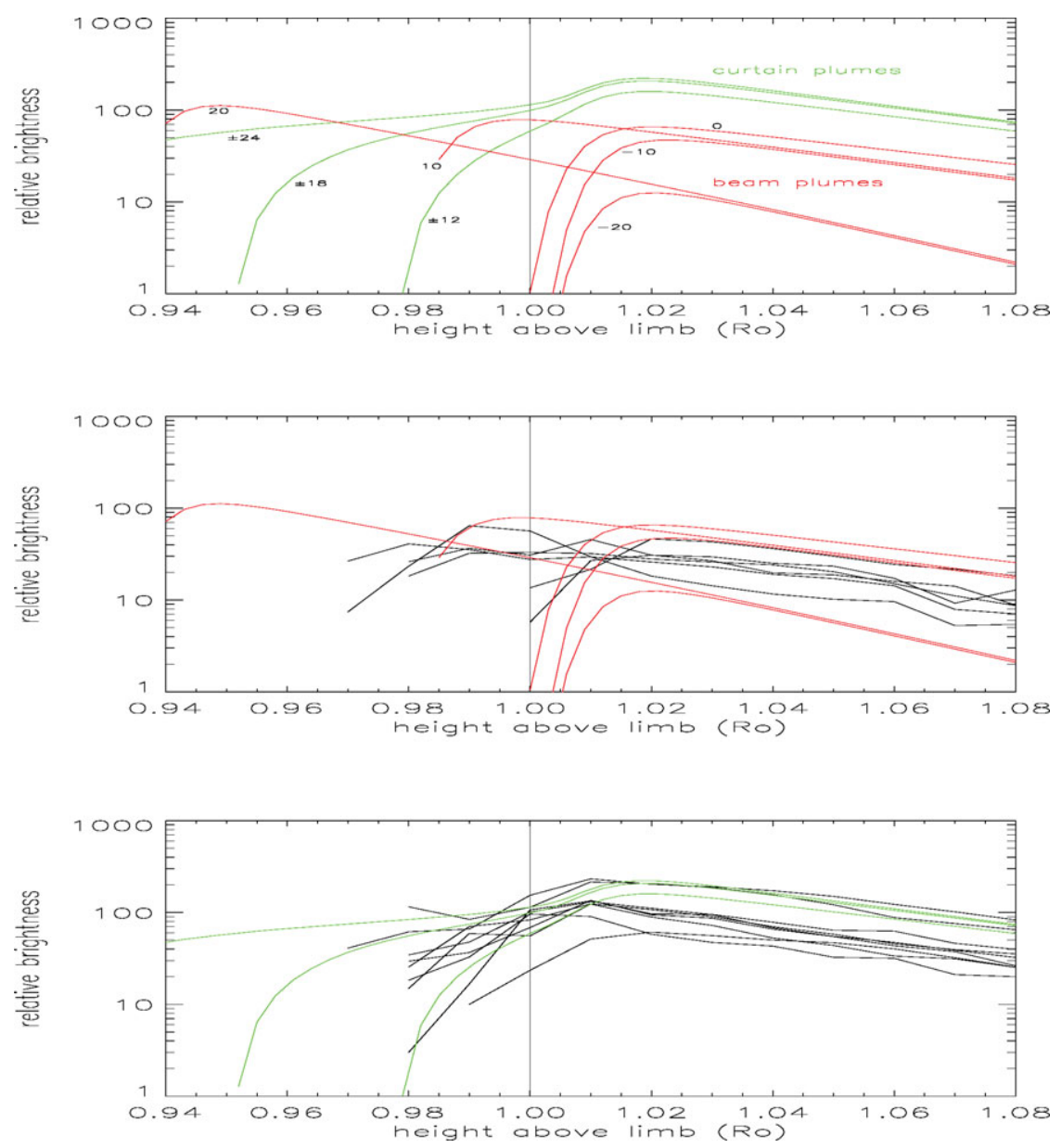

Figure 3. Showing (top) model calculations for the plume fall-off at the limb: beam plumes in red and curtain plumes in green, (middle) beam models with superposed observations, and (bottom) for curtain plumes. 


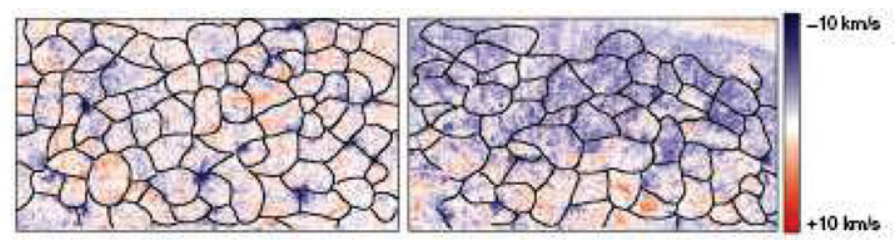

Figure 4. Dopplergram of Ne VIII in a coronal hole showing high outflow velocity at the boundaries of supergranule cells (Hasslet et al. 1999).
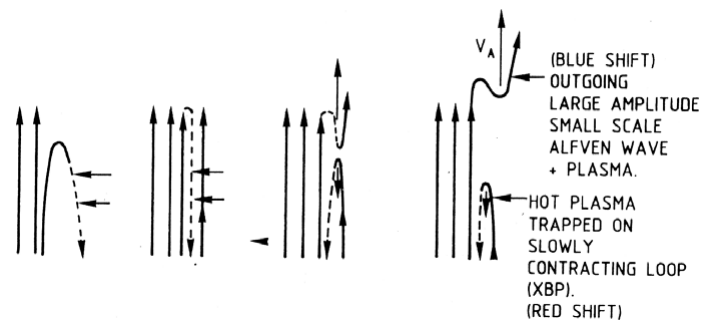

Figure 5. Schematic for the acceleration of fast wind from coronal hole regions (Axford \& McKenzie 1992).

straightforward. The only complication is that the $171 \AA$ emission will be absorbed by continuum in the cooler denser chromospheric layers. This effect becomes important when the tangential optical path passes close to the limb. It has the effect of smoothing out some of the curious sharp peaks that otherwise occur in the region $R_{\odot}=1.0$ to $R_{\odot}=1.01$. An effort has been made to include this in the model. Any uncertainties in this contribution can be shown to have a negligible effect on the interpretation of the data/modelling comparison. The two plume models are shown in red and in green in Fig. 3a. It can be seen that the curtain plumes are predicted to drop significantly in brightness as we cross inside the limb, by a factor extending from 3 to more than 10 , depending on the angular extent assumed for the curtain in the line-of-sight, shown here for $\pm 12^{\circ}, \pm 18^{\circ}$ and $\pm 24^{\circ}$. On the other hand, the beam plumes in red are predicted to continue to increase with decreasing radius, until they reach either their foot points on the front of the Sun, or the limb if behind the Sun. The two sets of curves are in principle mutually exclusive, and should allow discrimination between the observations. These curves, with brightness shown on a log scale, are in arbitrary units and can be slid vertically.

Since the simulations concern only the plumes and not the background corona, the observations need to be treated in a similar way. The observations used are those from all significant plumes seen in all four views shown in Fig. 1. These are first presented in the quantitative format of Fig. 2. Then for each trace, a series of Gaussian profiles has been fitted, in order to separate the plume from the corona and to correct for some blending of overlapping plumes. This fitting is extended onto the disk, only as far as we are confident for the continuity and identity of the plume (down to around 0.97 or $0.98 R_{\odot}$ ). The resulting radial brightness traces have been separated into the two categories indicated by the two models, and plotted in black in Figs 3b and c, overlaid with their respective model calculations. Their classification as beam or curtain is usually clear, with only one or two uncertain cases due to noise in the observations or in the Gaussian fitting. 


\section{Discussion and conclusions}

Limitations of space preclude a detailed discussion of the distribution of the two plume types. Here we state simply that the curtain plumes are normally much more abundant and they are always present when the solar conditions (viewing angle B, solar minimum) are optimised. The beam plumes are more variable and appear to be associated with small magnetic bright regions within the coronal hole. In the case of the 4 orthogonal views sampled here, it is the fourth on 14 Jun 1997 which contributes most of the beam plumes, corresponding with the increased bright-point activity seen in this image.

The ubiquitous nature of curtain plumes leads us to seek ubiquitous structures as their possible origin. Therefore we consider the supergranular network and its manifestations in the corona as the likely source. Linear structures associated with the cell boundaries, appear in chance alignment as the Sun rotates, leading to the observed plumes, with lifetimes of the order 1 or 2 days, due to the rotation.

It is worth recalling the study by Hassler et al. (1999), in which they showed a correlation between the boundaries of supergranular cells and high outflows in the fast solar wind (see Fig. 4). Their wind measurements used Ne VIII lines from SUMER, which are more likely typical of the upper transition region. The availability today of the EIS spectrometer on Hinode allows us to extend their measurements to true coronal temperatures. These studies are currently in progress. It is likely that curtain plumes are associated with the reconnection of emerging closed field loops with the dominant-polarity field in funnels at the boundaries of the cells, as first proposed by Axford \& McKenzie 1992. If this is true, they would have a direct association with the heating of the corona and the acceleration of the fast solar wind.

SOHO is a collaborative programme of ESA and NASA. Part of this work was carried out within the framework of the Coronal Plume study team (Leader, Klaus Wilhelm) of the International Space Science Institute (ISSI), Bern, Switzerland.

\section{References}

Axford, W. I. \& McKenzie, J. F. 1992, in: E. Marsch and R. Schwenn (eds.), Solar Wind Seven, Pergamon Press, P. 1

Gabriel, A. H., Bely-Dubau, F., \& Lemaire, P. 2003, ApJ, 589, 623

Gabriel, A. H., Abbo, L.,Bely-Dubau, F. Llebaria, A., \& Antonucci, E. 2005, ApJ, 635, L185

Hassler, D. M., Dammasch, I. E., Lemaire, P., Brekke, P., Curdt,W., Mason, H., Vial, J.-C., \& Wilhelm, K. 1999, Science, 283, 810

Noci, G., Kohl, J. L., \& Withbroe G. L. 1987, ApJ, 315, 706

Teriaca, L., Poletto, G., Romoli, M., \& Biesecker, D. A. 2003, ApJ, 588, 566

Wilhelm, K., Marsch, E., Dwivedi, B., Hassler, D. M., Lemaire, P. H., Gabriel, A. H., \& Huber, M. C. E. $1998, A p J, 500,1023$

Wilhelm, K. 2006, A\& A, 455, 697

\section{Discussion}

BOCHSLER: Is there an extra free parameter describing the relative orientation of curtain plumes to the line of sight? How are these orientation angles distributed?

GABRIEL: The curtain model is based on a volume emission intensity only 1 to $3 \%$ higher than the basic corona. Thus the brightness only reaches the observed level of $30 \%$ to $50 \%$ of the background when the line of sight reaches levels of $0.5 \mathrm{R}$ or higher. Only a narrow angle of 3 degrees around zero enables the plume to become detectable. 